less in tumour tissue (Abstract S91 Figure 1). Of the four SNPs studied two were associated with lung cancer; the associated allele of rs4588 is known to reduce macrophage activation and conferred an odds ratio (OR) of 3.04, whilst that of rs1544410 produces less stable VDR mRNA and conferred an OR of 4.10 of disease.

Conclusions Vitamin D deficiency is common in lung cancer, but reduced VDR in tumour tissue suggests that it is unlikely to be useful as an adjuvant treatment, as tumour tissue will not be able to respond to it. We have also confirmed the role of VDR polymorphisms in lung cancer, similar to other malignancies.

\section{S92 EPITHELIAL MESENCHYMAL TRANSITION OCCURS EARLIER THAN PREVIOUSLY THOUGHT IN THE DEVELOPMENT OF SQUAMOUS CELL CARCINOMA OF THE LUNG}

doi:10.1136/thx.2010.150938.43

${ }^{1} \mathrm{~N}$ M Cummings, ${ }^{2} \mathrm{D}$ M Rassl, ${ }^{2} \mathrm{H}$ C Boobier, ${ }^{3} \mathrm{~J}$ R Gosney, ${ }^{4} \mathrm{~A}$ G Nicholson, ${ }^{5} \mathrm{~L} D$ Sharples, ${ }^{1} \mathrm{R} C$ Rintoul. ${ }^{1}$ Department of Thoracic Oncology, Papworth Hospital, Cambridge, UK; ${ }^{2}$ Department of Pathology, Papworth Hospital, Cambridge, UK; ${ }^{3}$ Department of Histopathology, Royal Liverpool University Hospital, Liverpool, UK; ${ }^{4}$ Department of Histopathology, Royal Brompton Hospital, London, UK; ${ }^{5}$ MRC Biostatistics Unit, Cambridge, UK

Introduction Squamous cell carcinoma is believed to develop in a step-wise fashion from squamous metaplasia (SqMet), through lowand high-grade dysplasia (LGDys; HGDys) and carcinoma-in-situ (CIS) to invasive disease (InvSCC). Epithelial mesenchymal transition (EMT) is an important process by which epithelial cells shed their differentiated characteristics and acquire mesenchymal, fibroblast-like properties including increased motility and invasiveness. There is now convincing phenotypic, genetic and functional evidence that EMT plays a central role in carcinogenesis. To date, EMT is believed to occur during transition from pre-invasive disease to invasive disease. A hallmark of EMT is the down-regulation of E-cadherin, a cell adhesion molecule present in the plasma membrane of epithelial cells. We have examined the role of EMT during the progression of bronchial dysplasia.

Methods Using immunohistochemistry, 170 formalin-fixed paraffinembedded blocks from lung cancer biopsies and resection specimens were stained for epithelial markers E-cadherin and MNF116 and the mesenchymal marker S100A4. In each sample, areas of SqMet, LGDys, HGDys (incorporating CIS) and InvSCC were identified. Up to three representative areas were assessed for each lesion type present and an aggregate score assigned which took into account strength and extent of staining. Specimens were also stained for $\beta$-catenin which translocates from the membrane to the nucleus during EMT. One hundred cells per high power field were counted and the proportion of membranous, cytoplasmic and nuclear staining calculated.

Results There was a progressive loss of epithelial markers and a concurrent gain in S100A4 with increasing dysplasia. The switch from expression of epithelial to mesenchymal markers began as early as SqMet and reached significance for all three markers between LGDys and HGDys. Likewise, $\beta$-catenin showed translocation from membranous to cytoplasmic expression; this also reached significance between LGDys and HGDys. The difference in nuclear expression between groups did not reach significance.

Conclusions In order to improve lung cancer survival there is increasing interest in identifying early stage disease. The mechanisms underlying progression of bronchial dysplasia are poorly understood. Our results suggest that EMT is occurring much earlier in bronchial dysplasia than previously appreciated and this may have implications for the surveillance and treatment of pre-invasive disease.

\section{S93 IS BCL-2 IMPORTANT IN SMALL CELL LUNG CANCER?}

doi:10.1136/thx.2010.150938.44

${ }^{1} \mathrm{M}$ H Lawson, ${ }^{2} \mathrm{D}$ M Rassl, ' $\mathrm{S}$ L Vowler, ${ }^{2} \mathrm{~N}$ M Cummings, 'W J Howat, ' G Murphy, ${ }^{2} \mathrm{R}$ C Rintoul. ${ }^{1}$ CRUK Cambridge Research Institute, Cambridge, UK; ${ }^{2}$ Papworth Hospital NHS Foundation Trust, Cambridge, UK

Introduction Bcl-2 is an anti-apoptotic protein that has previously been associated with increased cell survival in Small Cell Lung Cancer (SCLC) in vitro. However, several immunohistochemical studies of Bcl-2 expression and survival using patient samples have produced conflicting results. We aimed to determine if $\mathrm{Bcl}-2$ expression has prognostic relevance in SCLC using a unique dedicated SCLC tissue microarray (TMA).

Methods The TMA was constructed using formal in fixed, paraffin embedded diagnostic biopsy samples (endobronchial biopsies, TBNA, CT-guided needle biopsies) from 203 patients diagnosed at Papworth Hospital (Cambridge, UK) between 1998 and 2005. 189 cases had cores of tissue on the completed TMA. There was some attrition due to the small size of individual biopsies. The TMA was stained for Bcl-2 using a standard antibody (DAKO clone 124) and the slides were scored by two readers for both extent and intensity of tumour cell staining. Data from the TMA were then correlated with clinical data. The results obtained were combined with those of previous studies in a meta-analysis.

Results 140 cases had tumour tissue on the TMA that could be evaluated for Bcl-2 staining. Patients with low intensity staining had better overall survival than those with high intensity staining in a Cox regression analysis (HR $0.55,95 \%$ CI 0.33 to $0.94, p=0.03$, $\mathrm{n}=117)$. The meta-analysis included 510 deaths in 673 cases and showed no significant effect of Bcl-2 on survival (HR 0.91, 95\% CI 0.74 to 1.09 ).

Conclusion This study equals the largest published study of $\mathrm{Bcl}-2$ expression in SCLC. Our data showed improved survival in SCLC patients with lower Bcl-2 expression, consistent with in vitro data and the rationale behind current on-going trials of $\mathrm{Bcl}-2$ antagonists in SCLC. However, our meta-analysis showed no overall effect and this may be due to differences in staining and scoring methods between the studies.

\section{S94 TWO NOVEL DETERMINANTS OF ETOPOSIDE RESISTANCE IN SMALL CELL LUNG CANCER}

doi:10.1136/thx.2010.150938.45

${ }^{1} \mathrm{M}$ H Lawson, ${ }^{2} \mathrm{~N} M$ Cummings, ${ }^{2} \mathrm{D} \mathrm{M}$ Rassl, ${ }^{1} \mathrm{R}$ Russell, ${ }^{1} \mathrm{~J} \mathrm{D}$ Brenton, ${ }^{2} \mathrm{R}$ C Rintoul, ${ }^{1} \mathrm{G}$ Murphy. ${ }^{1}$ CRUK Cambridge Research Institute, Cambridge, UK; ${ }^{2}$ Papworth Hospital NHS Foundation Trust, Cambridge, UK

Introduction Small Cell Lung Cancer (SCLC) typically responds well to initial chemotherapy with Etoposide and a platinum-containing agent. However survival is poor due to invariable relapse with chemoresistant disease. We used a unique series of SCLC cell lines (GLC-14, 16, 19), derived from a single patient at various timepoints during her treatment, to identify genes involved in Etoposide resistance. We then attempted to determine their functional role and validate their importance using patient specimens.

Methods The relationship of the cell lines to each other was confirmed using genomic methods. Genes whose expression pattern could explain the relative response of the cell lines to treatment with Etoposide were identified using cDNA microarray. These candidate Etoposide response genes were cloned from the cell line in which they were expressed at the highest level and transiently over-expressed in the cell line in which they were naturally expressed at the lowest level to determine whether this altered Etoposide resistance. Using 
immunohistochemistry, expression of the most promising candidates was examined using a tissue microarray (TMA).

Results The two most promising candidate genes were identified as DNA Polymerase $\beta$, a DNA repair enzyme and NKX2.2, a neuroendocrine transcription factor. Specific inhibition of DNA Polymerase $\beta$ with Pamoic Acid reduced the numbers of cells surviving treatment with Etoposide $(p=0.029)$ and increased the amount of DNA damage in the cells $(p<0.001)$. Stable overexpression of NKX2.2 significantly increased cell survival in response to Etoposide in two different SCLC cell lines. In keeping with this, we found that absence of nuclear staining for NKX2.2 in the TMA was an independent predictor of improved outcome in chemotherapy treated SCLC patients (HR $0.52,95 \%$ CI 0.33 to $0.82, p=0.005$ ).

Conclusion Using a biologically plausible model of in vivo acquired resistance to Etopoide we have identified two novel Etoposide resistance factors-DNA Polymerase $\beta$ and NKX2.2. Our in vitro data, in conjunction with the TMA results, provide justification for further prospective work to confirm the roles of these molecules in chemotherapy resistance in SCLC.

\section{Novel disease mechanisms in pulmonary arterial hypertension

S95 APPLICATION OF PROTEOMICS TO EXPLORE THE
PATHOBIOLOGY OF PULMONARY ARTERIAL HYPERTENSION

doi:10.1136/thx.2010.150938.46

V B Abdul-Salam, J Wharton, M R Wilkins, R J Edwards. Imperial College London, London, UK

Introduction and Objective Pulmonary arterial hypertension (PAH) is a poorly understood debilitating disorder which is characterised by increased resistance to the pulmonary blood flow caused by the remodelling of the pulmonary arteries. The objective of this study was to identify differentially expressed proteins in lungs of patients with PAH that may further help us understand the pathobiology of this disease.

Methods Label-free liquid chromatography tandem mass spectrometry was used to generate protein expression profiles from the lung homogenates of $8 \mathrm{PAH}$ patients and 8 Controls. Progenesis-MS software was then used to compare the relative levels of detected proteins.

Results After application of stringent criteria to confirm proteins identities and to detect differences, 27 proteins were found to be differentially expressed from a total of 363 proteins. Proteins that had increased expression in PAH lungs were mainly involved in cell growth, proliferation and cell metabolism. Examples of affected proteins included chloride intracellular channel 4 (CLIC4) and periostin which were both up-regulated and these findings were confirmed by immunoblotting. CLIC4 is known to play a vital role in angiogenesis and has also been implicated in transforming growth factor- $\beta$, vascular endothelial growth factor and bone morphogenetic protein receptor two signalling pathways. Periostin is an extracellular matrix protein considered to be involved in vascular injury responses and regulation of smooth muscle cell proliferation. Immunohistochemistry studies showed predominant localisation of CLIC4 in the endothelium of remodelled pulmonary arteries and in plexiform lesions while periostin was prominent in the airways and in the neointimal layer of the remodelled pulmonary arteries.

Conclusions Many proteins that may be involved in the pathogenesis of PAH have been discovered using label free proteomics. Examples include CLIC4 and periostin, which may be involved in the remodelling of vessels in $\mathrm{PAH}$.

\section{S96 NOVEL BIOMARKERS IN IDIOPATHIC PULMONARY ARTERIAL HYPERTENSION}

doi:10.1136/thx.2010.150938.47

C J Rhodes, J Wharton, L S Howard, J S R Gibbs, M R Wilkins. Imperial College London, London, UK

Background Circulatingosteopontin levels predict survival in right heart failure and lung tissue expression is increased in hypoxic animal models of pulmonary hypertension. Growth differentiation factor (GDF)-15 and red cell distribution width (RDW) are potential biomarkers in idiopathic pulmonary arterial hypertension (IPAH).

Objectives We assessed the prognostic value of these novel biomarkers, along with the more established $\mathrm{N}$-terminal pro-brain natriuretic peptide (NT-proBNP) and other clinical indices, in treatment-naïve and treated patients with IPAH.

Methods Plasmaosteopontin, GDF-15, and NT-proBNP levels and RDW were determined in 126 patients with IPAH, who were followed for $4.0 \pm 2.2$ years.

Results All four biomarkers correlated with WHO functional class and $6 \mathrm{~min}$ walk distance $(6 \mathrm{MWD})$ and cut-offs of osteopontin (53.4 ng/ml, p<0.05), GDF-15 (800 pg/ml)and RDW (15.65\%) predicted significant differences in survival in patients with IPAH (Abstract S96 Figure 1). Raised NT-proBNP levels also predicted significantly poorer survival $(p<0.01)$. A temporal increase in serially-measured levels ofGDF-15, osteopontin or RDW indicated poorer survival in IPAH. Patients with relatively low NT-proBNP levels (below median) who might normally be considered low risk, displayed higher mortality rates when GDF-15 levels or RDW were raised. Cox regression survival modelling confirmed RDW as a significant predictor of mortality, independent of established markers including NT-proBNP, 6MWD and WHO class. GDF-15, RDW and NT-proBNP predicted significant $(p<0.05)$ survival differences in patients with reduced exercise capacity (6MWD $<380 \mathrm{~m}$ ); median survival with low 6MWD and raised RDW $(>15.65 \%)$ was $<1.5$ year vs 3.6 year for low $6 \mathrm{MWD}$ irrespective of the RDW value.
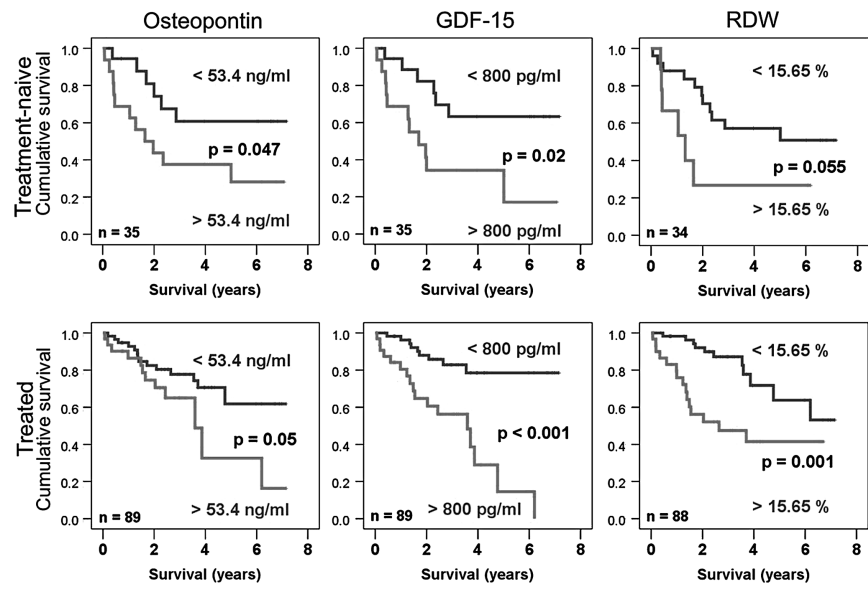

Abstract S96 Figure 1

Conclusions Osteopontin, GDF-15 and RDW all predicted survival in IPAH and were associated with poorer clinical status. Temporal changes in biomarkers appear to hold prognostic information. RDW and NT-proBNP may add the most value to $6 \mathrm{MWD}$ and WHO class. 